University of Nebraska - Lincoln

DigitalCommons@University of Nebraska - Lincoln

Architectural Engineering -- Faculty Publications

Architectural Engineering and Construction,

Durham School of

2010

Computational modeling of effects of alloying elements on elastic coefficients

Z. K. Liu

Pennsylvania State University

H. Zhang

Pennsylvania State University

S. Ganeshan

Pennsylvania State University

Y. Wang

Pennsylvania State University

S. N. Mathaudhu

U.S. Army Research Laboratory

Follow this and additional works at: https://digitalcommons.unl.edu/archengfacpub

Part of the Architectural Engineering Commons

Liu, Z. K.; Zhang, H.; Ganeshan, S.; Wang, Y.; and Mathaudhu, S. N., "Computational modeling of effects of alloying elements on elastic coefficients" (2010). Architectural Engineering -- Faculty Publications. 36. https://digitalcommons.unl.edu/archengfacpub/36

This Article is brought to you for free and open access by the Architectural Engineering and Construction, Durham School of at DigitalCommons@University of Nebraska - Lincoln. It has been accepted for inclusion in Architectural Engineering -- Faculty Publications by an authorized administrator of DigitalCommons@University of Nebraska Lincoln. 


\title{
Computational modeling of effects of alloying elements on elastic coefficients
}

\author{
Z.K. Liu, ${ }^{\mathrm{a}, *}$ H. Zhang, ${ }^{\mathrm{a}}$ S. Ganeshan, ${ }^{\mathrm{a}}$ Y. Wang ${ }^{\mathrm{a}}$ and S.N. Mathaudhu ${ }^{\mathrm{b}}$ \\ ${ }^{a}$ Department of Materials Science and Engineering, The Pennsylvania State University, University Park, PA 16802, USA \\ ${ }^{\mathrm{b}}$ Materials and Manufacturing Sciences Division, US Army Research Laboratory, Aberdeen Proving Ground, MD 21005-5069, USA
}

Received 9 December 2009; revised 15 March 2010; accepted 17 March 2010

Available online 19 March 2010

\begin{abstract}
Models for composition and temperature dependencies of single-crystal elastic stiffness coefficients are developed and applied to the $\mathrm{Al}_{12} \mathrm{Mg}_{17}$ and hexagonal closed-packed solution phases in the $\mathrm{Mg}-\mathrm{Al}$ system based on data from first-principles calculations. In combination with models for multi-phases, the bulk, shear, and Young's moduli of $\mathrm{Mg}-\mathrm{Al}$ alloys are predicted and compared with available experimental data in the literature. It is noted that both phase transition and grain boundary sliding may play important roles in the elastic coefficients as a function of temperature.
\end{abstract}

(c) 2010 Acta Materialia Inc. Published by Elsevier Ltd. All rights reserved.

Keywords: Elastic behavior; First-principles; Electron theory modeling; CALPHAD

\section{Introduction}

Computational methods in materials research offer powerful tools to provide fundamental understanding of the behaviors of materials and to support selection and design of materials that meet application requirements. With advances in computational science and information technology in recent years, materials research and development are shifting from the empirical approaches to the design to achieve optimal functionality. It is resulting in a new paradigm based on integrated computational-prediction and experimental-validation approaches [1,2]. Capabilities derived from the integration of first-principles calculations, mechanistic modeling, and simulations $[3,4]$.

Magnesium is the eighth most abundant element in the Earth's crust, the third most plentiful element dissolved in seawater, and the lightest structural metallic material, with a density of $1.741 \mathrm{~g} \mathrm{~cm}^{-3}$, particularly attractive for transportation applications such as automobiles and helicopters for weight reduction and higher fuel efficiency [5]. In previous works, we systematically studied thermodynamic properties of $\mathrm{Mg}$ alloys through integrating first-principles calculations and CALPHAD (CALculation of PHAse Diagram) modeling of relevant binary, ternary and multi-component systems [6]. Recently, we ventured into the development of approaches

\footnotetext{
$\bar{*}$ Corresponding author. E-mail: dr.liu@psu.edu
}

in predicating diffusion coefficients [7] and elastic constants [8]. In this paper, we develop models for composition and temperature dependences of single-crystal elastic stiffness coefficients, evaluate model parameters based on results from first-principles calculations, and combine them with the multi-phase models to predict bulk, shear, and Young's moduli of $\mathrm{Mg}$ alloys using the $\mathrm{Mg}-\mathrm{Al}$ binary system as the model system.

\section{Modeling methodology}

One successful modeling approach for properties of multi-component systems is the CALPHAD method. The CALPHAD approach was originally developed for modeling thermodynamic properties by integrating experimental phase equilibrium and thermochemical data $[4,9]$. The CALPHAD modeling begins with the development of models and the evaluation of parameters of unary and binary systems. By combining the constitutive binary systems with ternary experimental data, ternary interactions and properties of ternary phases are obtained. In this approach, properties of individual phases are modeled, covering the whole composition and temperature ranges, including experimentally uninvestigated regions. For pure elements and stoichiometric compounds, the most commonly used model is the one suggested by the Scientific Group Thermodata Europe (SGTE) [10]. The CALPHAD approach has been 
extended to model multi-component atomic mobility [11] and molar volume [12].

The CALPHAD modeling of properties in multicomponent systems can be written in a general form as follows for a phase $\alpha$ :

$\phi^{\alpha}={ }^{o} \phi^{\alpha}+\Delta \phi^{\alpha}$

where $\phi^{\alpha}$ is the property of the multi-component $\alpha$ phase, ${ }^{o} \phi^{\alpha}$ represents the mechanical mixing of individual species, and $\Delta \phi^{\alpha}$ denotes the interactions among the species. ${ }^{o} \phi^{\alpha}$ and $\Delta \phi^{\alpha}$ are commonly written as

$$
\begin{aligned}
{ }^{o} \phi^{\alpha}= & \sum x_{i}^{o} \phi_{i}^{\alpha} \\
\Delta \phi^{\alpha}= & \Delta \phi_{\text {conf }}^{\alpha}+\sum_{i} \sum_{j>i} x_{i} x_{j} \sum_{n=0}{ }^{n} \phi_{i, j}^{\alpha}\left(x_{i}-x_{j}\right)^{n} \\
& +\sum_{i} \sum_{j>i} \sum_{k>j} x_{i} x_{j} x_{k} \phi_{i j k}^{\alpha}
\end{aligned}
$$

where $x_{i}$ is the mole fraction of species $i,{ }^{o} \phi_{i}^{\alpha}$ the property of species $i$ in the structure of the $\alpha$ phase, $\Delta \phi_{\text {conf }}^{\alpha}$ the configurational contribution, and ${ }^{n} \phi_{i, j}^{\alpha}$ and ${ }^{n} \phi_{i, j}^{\alpha}$ are $n$th order binary and ternary interaction parameters. For thermodynamic modeling, $\Delta \phi_{\text {conf }}^{\alpha}$ is usually described by the ideal atomic configurational entropy of mixing, while for properties such as atomic mobility, molar volume, and elastic coefficients, there is no configurational contribution. One significant aspect of Eq. (2) is that all ${ }^{o} \phi_{i}^{\alpha}$ need to be in the same structure of the $\alpha$ phase. In case the stable structure of a component is not the same as the structure of the $\alpha$ phase, the difference of the properties between the two structures, called lattice stability in thermodynamic modeling, needs to be evaluated [9]. Efforts to predict thermodynamic lattice stability by first-principles calculations have been going on for many years [13]. New progresses have been made recently [14], and more works are needed in order to develop systematic approaches in predicting lattice stability for energy, atomic mobility, elastic coefficients, and other properties.

This paper aims to outline an approach for modeling of elastic coefficients as a function of temperature and composition and apply it to $\mathrm{Mg}$ alloys. The elastic energy of a crystal can be written as

$E_{e l}=\sum_{l=1}^{6} \sum_{m=1}^{6} \frac{1}{2} c_{l m} \varepsilon_{l} \varepsilon_{m}$

where $c_{l m}$ is a $6 \times 6$ elastic stiffness coefficient matrix, and $\varepsilon_{i}$ represents the strains. We can model $c_{l m}$ using the approach described by Eqs. (1)-(3) as follows:

$$
\begin{aligned}
c_{l m}={ }^{o} c_{l m}+\Delta c_{l m} \\
{ }^{o} c_{l m}=\sum_{i} x_{i}^{o} c_{l m}^{i} \\
\Delta c_{l m}=\sum_{i} \sum_{j>i} x_{i} x_{j} \sum_{n=0}{ }^{n} c_{l m}^{i, j}\left(x_{i}-x_{j}\right)^{n} \\
\quad+\sum_{i} \sum_{j>i} \sum_{k>j} x_{i} x_{i} x_{k} c_{l m}^{i j k}
\end{aligned}
$$

where ${ }^{o} c_{l m}^{i}$ is the elastic stiffness coefficient of species $i,{ }^{n} c_{l m}^{i, j}$ the $n$th order binary interaction parameters between species $i$ and $j$, and $c_{l m}^{i j k}$ the ternary interactions among species $i, j$, and $k$, all in the structure of the phase under consideration. In developing multi-component databases of elastic stiffness coefficients as a function of temperature and composition, it is crucial to first build a dataset for pure elements in common structure such as face-centered cubic (fcc), body-centered cubic (bcc), and hexagonal close-packed (hcp) similar to the SGTE thermodynamic data for pure elements [10], which is recently published by our group [15].

Let us first examine ${ }^{o} c_{l m}^{i}$, the elastic stiffness coefficients of a pure element or a stoichiometric compound $i$, as a function of temperature. Varshni [16] presented the following equation:

${ }^{o} c_{l m}^{i}={ }^{o} c_{l m}^{i 0}-\frac{S}{e^{t / T}-1}$

where ${ }^{o} c_{l m}^{i 0}, s$ and $t$ are constants. ${ }^{o} c_{l m}^{i 0}$ is the elastic stiffness coefficient at $0 \mathrm{~K}$. For higher temperatures, as vibrational energy changes linearly with respect to temperature, Lowrie and Gonas [17] and Slagle and McKinstry [18] presented polynomials in temperature as

${ }^{o} c_{l m}^{i}=a+b T+c T^{2}$

The similar equation was also tested by Varshni [16] with $b=0$ in Eq. (9) and used in the CALPHAD modeling approach, sometimes with additional higher-order terms [10]. We will test both Eqs. (8) and (9) in the present work.

For binary and ternary interaction parameters, let us follow the same approaches as in thermodynamic and atomic mobility modeling, i.e.

${ }^{n} c_{l m}^{i, j}={ }^{n} A_{l m}^{i, j}+{ }^{n} B_{l m}^{i, j} T+{ }^{n} C_{l m}^{i, j} T^{2}+\frac{{ }^{n} D_{l m}^{i, j}}{T^{2}}$

$c_{l m}^{i j k}=x_{i} I_{l m}^{i}+x_{j} I_{l m}^{j}+x_{k} I_{l m}^{k}$

with ${ }^{n} A_{l m}^{i, j},{ }^{n} B_{l m}^{i, j},{ }^{n} C_{l m}^{i, j},{ }^{n} D_{l m}^{i, j}, I_{l m}^{i}, I_{l m}^{j}$, and $I_{l m}^{k}$ being binary and ternary model parameters to be evaluated. For phases with more than one sublattice, Eqs. (6) and (7) can be rewritten in general as

$$
\begin{aligned}
{ }^{o} c_{l m}= & \sum_{e m}\left(\prod_{t} y_{i^{t}}^{t o} c_{l m}^{e m}\right) \\
\Delta c_{l m}= & \sum_{t} \prod_{s \neq t} y_{l^{s}}^{s} \sum_{i} \sum_{j>i} \sum_{n=0}{ }^{n} c_{l m}^{i^{t}, j^{t}: l^{s}}\left(y_{i}^{t}-y_{j}^{t}\right)^{n} \\
& +\sum_{t} \prod_{s \neq t} y_{l^{s}}^{s} \sum_{i} \sum_{j>i} \sum_{k>j} y_{i}^{t} y_{j}^{t} y_{k}^{t} c_{l m}^{i^{t} j^{t} k^{t}: l^{s}} \\
& +\sum_{t} \sum_{u>t} \prod_{s \neq t \neq u} y_{l^{s}}^{s} \sum_{i} \sum_{j>i} \sum_{k} \sum_{l>k} y_{i}^{t} y_{j}^{t} y_{k}^{u} y_{l}^{u} c_{l m}^{i^{t} j^{t}: k^{s} l^{s}}
\end{aligned}
$$

where ${ }^{o} c_{l m}^{e m}$ is the elastic stiffness coefficient of the endmember. The first and second terms in Eq. (13) represent the binary and ternary interaction parameters in each sublattice, while the third term describes the interaction between two sublattices [4,19].

There are two classic approaches to calculating the polycrystal elastic properties in terms of the single-crystal elastic coefficients: one is Voigt's approach for the upper bound based on the uniform strain, and the other is Reuss's approach for the lower bound based on the 
uniform stress (see e.g. Ref. [20]). According to Hill's empirical average [21], the bulk $\left(B_{H}\right)$, shear $\left(G_{H}\right)$ and Young's $\left(E_{H}\right)$ moduli are calculated as $B_{H}=\left(B_{V}+\right.$ $\left.B_{R}\right) / 2, G_{H}=\left(G_{V}+G_{R}\right) / 2$, and $E_{H}=\left(9 B_{H} G_{H}\right) /\left(3 B_{H}+\right.$ $G_{H}$ ), with subscripts $V$ and $R$ representing Voigt and Reuss approaches, respectively. By employing a variational approach and considering the crystal anisotropy, Hashin and Shtrikman [22] developed upper and lower bounds as a function of orientation averages, which are tighter than the Voigt-Reuss approaches. Further development includes extensions to consider the twopoint and high-order correlation functions and strong textured grains [23]. For simplicity, we will use Hill's average in the present work.

Furthermore, Hashin and Shtrikman [24] considered multi-phase materials as mechanical mixtures of a number of different isotropic and homogeneous elastic phases. With the smallest bulk and shear moduli of phases denoted by $B_{1}$ and $G_{1}$, and the largest by $B_{n}$ and $G_{n}$, the bounds of effective bulk and shear moduli, with $B_{1}^{*}$ and $G_{1}^{*}$ for the lower bounds and $B_{n}^{*}$ and $G_{n}^{*}$ for the upper bounds, are obtained as follows, respectively:

$B_{i}^{*}=B_{i}+\frac{A_{i}}{1+\alpha_{i} A_{i}}$

$G_{i}^{*}=G_{i}+\frac{S_{i}}{2+2 \beta S_{i}}$

with $\alpha_{i}=\frac{3}{3 B_{i}+4 G_{i}}, A_{i}=\sum_{j \neq 1} \frac{V_{j}}{\frac{1}{B_{j}-B_{i}}}, \beta_{i}=-\frac{3\left(B_{i}+2 G_{i}\right)}{5 G_{i}\left(3 B_{i}+4 G_{i}\right)}$ and $S_{i}=\sum_{j \neq 1} \frac{V_{j}}{2\left(G_{j}-G_{i}\right)}-\beta_{i}$ where $V_{j}$ is the volume fraction of phase $j$, and $i=1$ and $n$ for the lower and upper bonds, respectively. These bounds have been widely used for composite materials in the literature [23].

\section{Elastic coefficients by first-principles calculations}

The elastic coefficients can be obtained using the efficient strain-stress method [8]. Consider a crystal with lattice vectors $\boldsymbol{R}$ subject to a set of strains, $\boldsymbol{\varepsilon}=\left(\varepsilon_{1}, \varepsilon_{2}\right.$, $\left.\varepsilon_{3}, \varepsilon_{4}, \varepsilon_{5}, \varepsilon_{6}\right)$, with $\varepsilon_{1}, \varepsilon_{2}$, and $\varepsilon_{3}$ being the normal strains and $\varepsilon_{4}, \varepsilon_{5}$ and $\varepsilon_{6}$ the shear strains. For small shape change due to strains $\varepsilon$, the deformed lattice vectors can be approximated as follows:

$$
\boldsymbol{R}_{\text {def }}=\boldsymbol{R}\left(\begin{array}{ccc}
1+\varepsilon_{1} & \varepsilon_{6} / 2 & \varepsilon_{5} / 2 \\
\varepsilon_{6} / 2 & 1+\varepsilon_{2} & \varepsilon_{4} / 2 \\
\varepsilon_{5} / 2 & \varepsilon_{4} / 2 & 1+\varepsilon_{3}
\end{array}\right)
$$

The corresponding set of stresses, $\boldsymbol{\sigma}=\left(\sigma_{1}, \sigma_{2}, \sigma_{3}, \sigma_{4}, \sigma_{5}\right.$, $\sigma_{6}$ ) for the deformed crystal, is obtained directly from the output of the first-principles calculations, which is more accurate than the stresses calculated from derivatives of the work done. Based on the general Hooke's law, the elastic stiffness coefficients are calculated by

$\mathrm{c}=\boldsymbol{\varepsilon}^{-1} \boldsymbol{\sigma}$

where $\varepsilon^{-1}$ represents the (pseudo)inverse of the sets of strains. The number of independent elastic stiffness constants can be reduced based on crystal symmetries [25]. The bulk, shear and Young's moduli can be derived from the calculated first-principles elastic stiffness constants.
The temperature dependence of elastic stiffness coefficients can be obtained through first-principles calculations with the quasi-harmonic phonon approximation. The Helmholtz energy of a crystal can be obtained from first-principles calculations by considering the static energy at $0 \mathrm{~K}$, the lattice vibrational free energy of the lattice ions, and the thermal electronic contribution [26]. The equilibrium volume at a given temperature, $V(T)$, can be computed through the derivative of Helmholtz energy to volume which defines the external pressure. As noted by Ledbetter [27], the temperature dependence of elastic stiffness coefficients is primarily due to the volume expansion with temperature increasing. By calculating the elastic stiffness coefficients at various volumes, the following equation can be used to evaluate their temperature dependence [28]:

$c_{i j}(V) \stackrel{V(T)}{\Longleftrightarrow} c_{i j}(T)$

It should be noted that the elastic stiffness coefficients thus obtained are under isothermal conditions. On the other hand, when the elastic stiffness coefficients are measured by resonant vibrations, the system may be viewed as adiabatic because elastic waves travel faster than heat diffuses, and the deformation due to the elastic waves is thus a constant-entropy (isentropic) process. Consequently, the isothermal elastic stiffness coefficients, $c_{i j}^{T}$, are different from the adiabatic or isentropic elastic stiffness coefficients, $c_{i j}^{S}$, with their thermodynamic relations discussed by Davies [29] as follows:

$$
\begin{aligned}
c_{i j}^{S} & =c_{i j}^{T}+\frac{T \lambda_{i} \lambda_{j}}{\rho C_{\varepsilon}} \\
\lambda_{i} & =\left(\frac{\partial S}{\partial \varepsilon_{i}}\right)_{T}=-\left(\frac{\partial \sigma_{i}}{\partial T}\right)_{\varepsilon}=-\frac{\partial^{2} F}{\partial T \partial \varepsilon_{i}} \\
& =-\sum_{j}\left(\frac{\partial \sigma_{i}}{\partial \varepsilon_{j}}\right)_{T}\left(\frac{\partial \varepsilon_{j}}{\partial T}\right)_{\sigma}=-\sum_{j} \alpha_{j} c_{i j}^{T}
\end{aligned}
$$

where $\rho$ is the density, $C_{\varepsilon}$ the heat capacity under constant strain, and $\alpha_{j}$ the thermal expansion. For a stable phase, the second derivative of its Helmholtz energy in Eq. (20) is positive, and therefore both $\lambda_{i}$ and $\lambda_{j}$ are negative. This results in $c_{i j}^{T}<c_{i j}^{S}$.

For solution phases or compounds with homogeneity ranges, the supercell method can be employed. For dilute solutions, one solvent atom is substituted by one solute atom in a supercell. For concentrated solutions, the special quasi-random structure (SQS) approach $[30,31]$ can be employed with their pair and multiple correlation functions as close as possible to random solutions. The first-principles approaches described above can be directly applied to the SQS supercells to obtain elastic stiffness coefficients of solid solution phases as a function of temperature.

\section{Elastic coefficients in Mg alloys}

Based on our efficient strain-stress method [8] as outlined previously, the elastic coefficients of binary $\mathrm{Mg}-\mathrm{X}$ dilute hcp solutions and binary compounds at $0 \mathrm{~K}$ have 
been predicted from first-principles calculations $[32,33]$. In the present paper, we focus on the elastic coefficients of the $\mathrm{Mg}-\mathrm{Al}$ system. In the $\mathrm{Mg}-\mathrm{Al}$ system, the $\mathrm{Al}_{12} \mathrm{Mg}_{17}$ intermetallic compound is in equilibrium with the hcp solid solution phase [34]. Zhang et al. [35] recently predicted the elastic coefficients of pure hcp-Mg and $\mathrm{Al}_{12} \mathrm{Mg}_{17}$ as a function of temperature. The results from first-principles calculations $[33,35]$ are used to evaluate model parameters based on Eqs. (8) and (9) as shown in Table 1 for $\alpha$-hcp of pure $\mathrm{Mg}$ and Table 2 for $\gamma-\mathrm{Al}_{12} \mathrm{Mg}_{17}$ with the coefficient of determination $\left(R^{2}\right)$ between 0.95 and 1 (also true for parameters in all other tables). All isentropic elastic stiffness coefficients are calculated in the present work using equations discussed earlier. It is found that there is no need to include the parameter $c$ in Eq. (9) for either $\alpha$ or $\gamma$ phase. The parameters ${ }^{n} C_{l m}^{i, j}$ and ${ }^{n} D_{l m}^{i, j}$ in Eq. (10) are not needed either. It should be mentioned that the expressions for elastic compliance coefficients are quite complex for non-cubic structures, and so are their $B_{R}$ and $G_{R}$. Consequently, they are fitted to their values using the simple formula as shown in Eqs. (8) and (9). The same is true for $E_{H}$ when Eq. (8) is used.

To evaluate the effect of alloying elements, the temperature dependences of elastic stiffness coefficients of with 2.78 at.\% $\mathrm{Al}$ are obtained from first-principles calculations in the present work using a supercell of 36 atoms with one being $\mathrm{Al}$ and 35 being $\mathrm{Mg}$ as in Ref. [33], and the model parameters are listed in Table 3. In order to model the elastic coefficients in the whole composition range as shown in Eq. (6), the elastic stiffness coefficients of hcp-Al are obtained from first-principles calculations in the present work with parameters listed in Table 4. Based on these data, the interaction parameters between $\mathrm{Mg}$ and $\mathrm{Al}$ in $\alpha$ are evaluated from Eq. (10) with only zero-order interaction parameters considered for each elastic stiffness coefficient due to the only data point at 2.78 at.\% $\mathrm{Al}$ (see Table 5). The elastic stiffness coefficients of the Mg-Al-hcp solid solution as a function of temperature and composition are thus represented by the following equation:

$$
\begin{aligned}
c_{l m}^{h c p}= & x_{A l}{ }^{o} c_{l m}^{A l-h c p}+x_{M g}{ }^{o} c_{l m}^{M g-h c p} \\
& +x_{A l} x_{M g}\left({ }^{0} A_{l m}^{A l, M g}+{ }^{0} B_{l m}^{A l, M g} T\right)
\end{aligned}
$$

with ${ }^{o} C_{l m}^{M g-h c p}$ from Table $1,{ }^{o} C_{l m}^{A l-h c p}$ from Table 4, and ${ }^{0} A_{l m}^{A l, M g}$ and ${ }^{0} B_{l m}^{A l, M g}$ from Table 5.

For a two-phase mixture, Eqs. (14) and (15) reduce to

$$
\begin{aligned}
& B_{i}^{*}=B_{i}+\frac{V_{j}\left(B_{j}-B_{i}\right)\left(3 B_{i}+4 G_{i}\right)}{3 B_{i}+4 G_{i}+3 V_{i}\left(B_{j}-B_{i}\right)} \\
& G_{i}^{*}=G_{i}+\frac{5 G_{i} V_{j}\left(G_{j}-G_{i}\right)\left(3 B_{i}+4 G_{i}\right)}{5 G_{i}\left(3 B_{i}+4 G_{i}\right)+6 V_{i}\left(G_{j}-G_{i}\right)\left(B_{i}+2 G_{i}\right)}
\end{aligned}
$$

For the lower bonds, $B_{i}$ and $G_{i}$ are from the phase with lower values, and $B_{j}$ and $G_{j}$ from the phase with higher values. For the upper bonds, $B_{i}$ and $G_{i}$ are from the phase with higher values, and $B_{j}$ and $G_{j}$ from the phase with lower values. We apply the above equations to binary $\mathrm{Mg}-\mathrm{Al}$ binary alloys with 3 and 9 wt.\% $\mathrm{Al}$, respectively, in analogy to the AZ31 and AZ91 alloys. The mole fractions of phases as a function of temperature are obtained from thermodynamic calculations [34].

For simplicity, we approximate the elastic properties of the off-stoichiometric $\gamma$ phase with those of the stoichiometric one as its composition in equilibrium with the hcp solution phase is almost constant with respect to temperature. The isothermal and isentropic bulk $\left(B_{H}\right)$, shear $\left(G_{H}\right)$, and Young's $\left(E_{H}\right)$ moduli of $\mathrm{Mg}-$ 3 wt.\% Al and $\mathrm{Mg}-9$ wt.\% Al are shown in Figures 1 and 2, respectively, along with experimental, isentropic shear $\left(G_{H}\right)$, and Young's $\left(E_{H}\right)$ moduli data of the AZ31 (Mg-3 wt.\% Al-1 wt.\% Zn) and AZ91 (Mg9 wt.\% Al-1 wt.\% $\mathrm{Zn}$ ) alloys available in the literature

\begin{tabular}{|c|c|c|c|c|c|c|c|c|c|c|}
\hline & \multicolumn{2}{|l|}{$c_{i j}^{T}$} & \multicolumn{2}{|l|}{$c_{i j}^{S}$} & \multicolumn{3}{|l|}{$c_{i j}^{T}$} & \multicolumn{3}{|l|}{$c_{i j}^{S}$} \\
\hline & $a$ & $b$ & $a$ & $b$ & ${ }^{o} c_{l m}^{i 0}$ & $s$ & $t$ & ${ }^{o} c_{l m}^{i 0}$ & $s$ & $t$ \\
\hline$c_{11}$ & 59.583 & $-2.023 \times 10^{-2}$ & 59.248 & $-1.158 \times 10^{-2}$ & 58.987 & 2.396 & 112.892 & 58.987 & 0.361 & 23.078 \\
\hline$c_{12}$ & 29.643 & $-1.083 \times 10^{-2}$ & 29.305 & $-6.354 \times 10^{-3}$ & 28.872 & 4.738 & 356.350 & 28.872 & 0.696 & 112.164 \\
\hline$c_{13}$ & 20.658 & $-2.597 \times 10^{-3}$ & 20.478 & $-5.969 \times 10^{-4}$ & 20.431 & 1.655 & 464.729 & 20.431 & 0.017 & 31.736 \\
\hline$c_{33}$ & 64.419 & $-1.921 \times 10^{-2}$ & 63.934 & $-1.415 \times 10^{-2}$ & 63.102 & 7.927 & 343.544 & 63.102 & 1.468 & 105.147 \\
\hline$c_{44}$ & 17.656 & $-3.019 \times 10^{-3}$ & 17.656 & $-3.019 \times 10^{-3}$ & 17.610 & 0.185 & 60.981 & 17.610 & 0.185 & 60.981 \\
\hline$B_{H}$ & 36.167 & $-1.013 \times 10^{-2}$ & 35.883 & $-6.761 \times 10^{-3}$ & 35.318 & 5.511 & 413.917 & 35.568 & 0.732 & 107.313 \\
\hline$G_{H}$ & 17.565 & $-5.021 \times 10^{-3}$ & 17.535 & $-4.720 \times 10^{-3}$ & 17.447 & 0.555 & 102.892 & 17.494 & 0.0843 & 17.854 \\
\hline$E_{H}$ & 45.352 & $-1.293 \times 10^{-2}$ & 45.236 & $-1.145 \times 10^{-2}$ & 44.923 & 2.101 & 146.861 & 45.089 & 0.325 & 27.715 \\
\hline
\end{tabular}
measured by means of resonant vibrations. It is noted that the predicted values of isothermal shear modules

Table 1. Two sets of model parameters for hcp-Mg based on Eqs. (8) and (9), respectively, evaluated from first-principles calculations in Ref. [35].

Table 2. Two sets of model parameters for $\mathrm{Al}_{12} \mathrm{Mg}_{17}$ based on Eqs. (8) and (9), respectively, evaluated from first-principles calculations in Ref. [35].

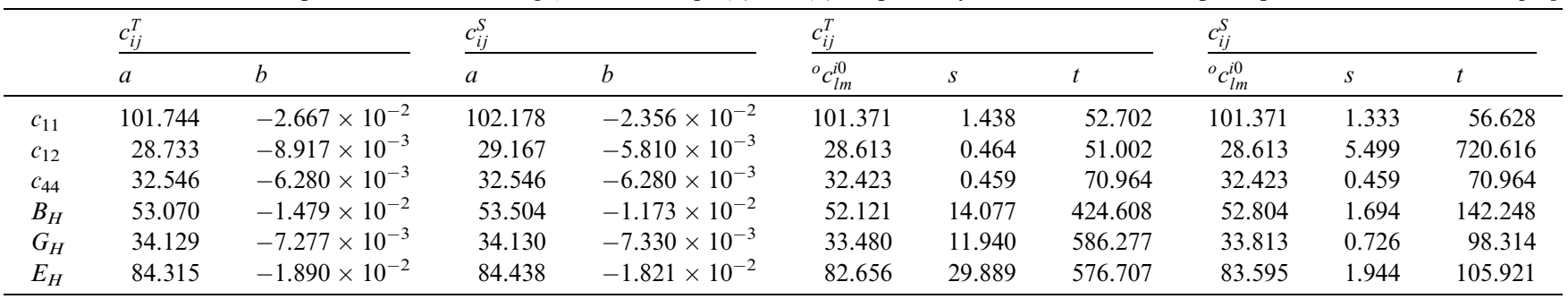


Table 3. Model parameters for Mg-2.78 at.\% Al-hcp based on Eqs. (8) and (9), respectively, evaluated from present first-principles calculations.

\begin{tabular}{|c|c|c|c|c|c|c|c|c|c|c|}
\hline & \multicolumn{2}{|l|}{$c_{i j}^{T}$} & \multicolumn{2}{|l|}{$\underline{c_{i j}^{S}}$} & \multicolumn{3}{|l|}{$\underline{c_{i j}^{T}}$} & \multicolumn{3}{|l|}{$\underline{c_{i j}^{S}}$} \\
\hline & $a$ & $b$ & $a$ & $b$ & ${ }^{o} c_{l m}^{i 0}$ & $s$ & $t$ & ${ }^{o} c_{l m}^{i 0}$ & $s$ & $t$ \\
\hline$c_{11}$ & 57.874 & -0.0171 & 57.109 & $-8.551 \times 10^{-3}$ & 58.325 & -1.096 & -64.852 & 58.325 & -0.929 & -103.068 \\
\hline$c_{12}$ & 30.639 & -0.0102 & 30.129 & $-4.501 \times 10^{-3}$ & 31.175 & -0.352 & -31.559 & 31.175 & -0.970 & -197.049 \\
\hline$c_{13}$ & 20.161 & -0.0072 & 19.6337 & $-1.311 \times 10^{-3}$ & 21.200 & -0.732 & -83.307 & 21.200 & -0.754 & -473.362 \\
\hline$c_{33}$ & 71.527 & -0.0218 & 70.710 & $-1.267 \times 10^{-2}$ & 71.250 & -0.837 & -41.311 & 71.250 & -0.139 & -10.794 \\
\hline$c_{44}$ & 14.866 & -0.0042 & 14.866 & $-4.204 \times 10^{-3}$ & 14.400 & -0.200 & -69.827 & 14.400 & -0.200 & -69.827 \\
\hline$B_{H}$ & 36.557 & $-1.162 \times 10^{-2}$ & 35.969 & $-4.891 \times 10^{-3}$ & 35.805 & 4.037 & 296.973 & 35.886 & 0.179 & 36.494 \\
\hline$G_{H}$ & 16.014 & $-4.217 \times 10^{-3}$ & 16.347 & $-3.593 \times 10^{-3}$ & 15.180 & 1.992 & 498.274 & 15.774 & 1.751 & 451.816 \\
\hline$E_{H}$ & 41.851 & $-1.142 \times 10^{-2}$ & 42.588 & $-8.669 \times 10^{-3}$ & 39.892 & 5.222 & 466.589 & 41.253 & 3.974 & 416.768 \\
\hline
\end{tabular}

Table 4. Model parameters for hcp-Al based on Eqs. (8) and (9), respectively, evaluated from present first-principles calculations.

\begin{tabular}{|c|c|c|c|c|c|c|c|c|c|c|}
\hline & \multicolumn{2}{|l|}{$c_{i j}^{T}$} & \multicolumn{2}{|l|}{$c_{i j}^{S}$} & \multicolumn{3}{|l|}{$c_{i j}^{T}$} & \multicolumn{3}{|l|}{$c_{i j}^{S}$} \\
\hline & $a$ & $b$ & $\bar{a}$ & $b$ & $\overline{{ }^{o} c_{l m}^{i 0}}$ & $s$ & $t$ & $\overline{{ }^{o} c_{l m}^{i 0}}$ & $s$ & $t$ \\
\hline$c_{11}$ & 103.024 & $-4.290 \times 10^{-2}$ & 101.578 & $-2.907 \times 10^{-2}$ & 101.333 & 7.353 & 158.110 & 101.333 & $-8.111 \times 10^{-3}$ & -0.276 \\
\hline$c_{12}$ & 67.287 & $-2.402 \times 10^{-2}$ & 65.641 & $-1.227 \times 10^{-2}$ & 66.021 & 6.949 & 249.814 & 66.021 & -0.184 & -14.465 \\
\hline$c_{13}$ & 52.379 & $-1.819 \times 10^{-2}$ & 50.742 & $-6.668 \times 10^{-3}$ & 51.403 & 5.430 & 256.279 & 51.403 & -0.583 & -80.723 \\
\hline$c_{33}$ & 108.921 & $-4.751 \times 10^{-2}$ & 107.129 & $-3.448 \times 10^{-2}$ & 106.639 & 11.531 & 214.719 & 106.639 & 0.181 & 5.347 \\
\hline$c_{44}$ & 4.007 & $-8.601 \times 10^{-3}$ & 4.007 & $-8.601 \times 10^{-3}$ & 3.870 & 0.325 & 38.173 & 3.870 & 0.325 & 38.173 \\
\hline$B_{H}$ & 73.229 & $-2.818 \times 10^{-2}$ & 71.615 & $-1.598 \times 10^{-2}$ & 71.370 & 7.794 & 248.014 & 71.512 & 0.221 & 13.794 \\
\hline$G_{H}$ & 14.705 & $-1.020 \times 10^{-2}$ & 14.740 & $-9.599 \times 10^{-3}$ & 14.359 & 1.363 & 125.674 & 14.549 & 0.408 & 42.348 \\
\hline$E_{H}$ & 41.347 & $-2.730 \times 10^{-2}$ & 41.382 & $-2.397 \times 10^{-2}$ & 40.331 & 4.262 & 141.213 & 40.867 & 1.365 & 51.571 \\
\hline
\end{tabular}

Table 5. Model parameters for the Mg-Al-hcp solid solution based on Eq. (10) with the hcp-Mg and hcp-Al from Eqs. (8) and (9), respectively.

\begin{tabular}{|c|c|c|c|c|c|c|c|c|}
\hline & \multicolumn{2}{|c|}{${ }^{o} c_{l m}^{T}=a+b T$} & \multicolumn{2}{|c|}{${ }^{o} c_{l m}^{S}=a+b T$} & \multicolumn{2}{|c|}{${ }^{o} c_{l m}^{T}=a+b T$} & \multicolumn{2}{|c|}{${ }^{o} c_{l m}^{S}=a+b T$} \\
\hline & $\overline{{ }^{o} A_{l m}^{i, j}}$ & ${ }^{o} B_{l m}^{i, j}$ & $\overline{{ }^{o} A_{l m}^{i, j}}$ & ${ }^{o} B_{l m}^{i, j}$ & $\overline{{ }^{o} A_{l m}^{i, j}}$ & ${ }^{o} B_{l m}^{i, j}$ & $\overline{{ }^{o} A_{l m}^{i, j}}$ & ${ }^{o} B_{l m}^{i, j}$ \\
\hline$c_{11}$ & -108.133 & 0.138 & -122.956 & 0.131 & -125.534 & 0.173 & -118.231 & 0.275 \\
\hline$c_{12}$ & -1.735 & 0.036 & -6.776 & $7.492 \times 10^{-2}$ & -4.561 & $4.248 \times 10^{-2}$ & -0.682 & $6.556 \times 10^{-2}$ \\
\hline$c_{13}$ & -51.078 & -0.155 & -62.475 & $-2.027 \times 10^{-2}$ & -34.032 & -0.193 & -54.297 & $-2.260 \times 10^{-2}$ \\
\hline$c_{33}$ & 218.147 & -0.082 & 207.164 & $7.586 \times 10^{-2}$ & 163.985 & $5.286 \times 10^{-2}$ & 223.035 & $5.155 \times 10^{-2}$ \\
\hline$c_{44}$ & -89.554 & -0.038 & -89.554 & $-3.881 \times 10^{-2}$ & -112.362 & $1.188 \times 10^{-2}$ & -112.362 & $1.188 \times 10^{-2}$ \\
\hline$B_{H}$ & -25.642 & -0.036 & -33.557 & $7.891 \times 10^{-2}$ & -26.138 & $-1.501 \times 10^{-2}$ & -32.233 & $7.706 \times 10^{-2}$ \\
\hline$G_{H}$ & -55.233 & 0.032 & -41.235 & $4.686 \times 10^{-2}$ & -74.014 & 0.079 & -48.328 & $5.725 \times 10^{-2}$ \\
\hline$E_{H}$ & -125.517 & 0.064 & -94.355 & $6.683 \times 10^{-2}$ & -168.756 & 0.180 & -110.313 & 0.142 \\
\hline
\end{tabular}

in both alloys are well in line with the experimental isentropic ones at low temperatures, indicating the potentially small corrections between isothermal and isentropic shear moduli at low temperatures. As can be seen, the correction becomes more significant at high temperatures.

In AZ31, Watanabe et al. [36] suggested that the drop in elastic constants in fine-grained hot-rolled samples at above $400-423 \mathrm{~K}$ as a result of the onset of grain boundary sliding. The current calculations indicate that this drop may also be affected by the decrease of the amount

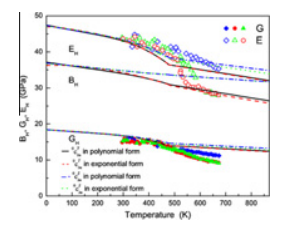

Figure 1. Calculated bulk $\left(B_{H}\right)$, shear $\left(G_{H}\right)$, and Young's $\left(E_{H}\right)$ moduli of $\mathrm{Mg}-3 \mathrm{wt} . \% \mathrm{Al}$ as a function of temperature in comparison with the experimental data of AZ31 [36] with the diamond symbols from solution treated and the triangle and square symbols from plates and sheets after hot rolling, respectively. of $\gamma-\mathrm{Al}_{12} \mathrm{Mg}_{17}$. At higher temperatures, Watanabe et al. [36] observed significantly lower shear and Young's moduli in the hot-rolled plate (average grain size of $51 \mu \mathrm{m}$ ) and sheet (average grain size of $11 \mu \mathrm{m}$ ) than those in the sample solution-treated at $686 \mathrm{~K}$ for $48 \mathrm{~h}$ (average grain size of $150 \mu \mathrm{m}$ ). This difference is more dramatic at temperatures higher than $475 \mathrm{~K}$ where the alloy is in the single $\alpha$-hcp phase region at equilibrium, keeping in mind that the phase relation in AZ31 is slightly different from the $\mathrm{Mg}-3 \mathrm{wt} . \% \mathrm{Al}$. The significantly lower shear and Young's moduli are likely due

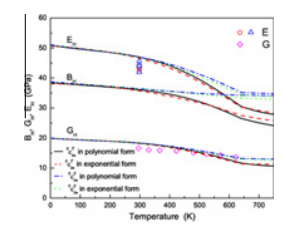

Figure 2. Calculated bulk $\left(B_{H}\right)$, shear $\left(G_{H}\right)$, and Young's $\left(E_{H}\right)$ moduli of $\mathrm{Mg}-9 \mathrm{wt} . \% \mathrm{Al}$ as a function of temperature in comparison with experimental Young's modulus $(\boldsymbol{O})$ [37], ( $\Delta)$ [38], and shear modulus $(\diamond)[39]$ of the AZ91 alloy. 
to the grain boundary sliding as suggested by the authors [36]. On the other hand, the kinks on the shear and Young's moduli are less visible for the solution-treated sample. This is probably because the sample did not reach equilibrium at the temperatures of measurements as the holding time at those temperatures was not mentioned and was probably very short. As most $\gamma$ $\mathrm{Al}_{12} \mathrm{Mg}_{17}$ particles are typically on grain boundaries, larger grain sizes imply larger sizes of $\gamma-\mathrm{Al}_{12} \mathrm{Mg}_{17}$ particles for a given alloy composition and thus lower dissolution rates of $\gamma-\mathrm{Al}_{12} \mathrm{Mg}_{17}$ and longer time to reach equilibrium. The interplay of $\gamma-\mathrm{Al}_{12} \mathrm{Mg}_{17}$ dissolution and grain boundary sliding jointly determines the elastic properties at high temperatures.

The comparison for AZ91 is shown in Figure 2. The experimental shear modulus is lower than the predicted one at low temperatures, but higher at high temperatures. This is probably due to the higher amount of $\gamma$ $\mathrm{Al}_{12} \mathrm{Mg}_{17}$ than its equilibrium amount at temperatures of measurements as there is not enough time for the alloy to reach equilibrium.

\section{Summary}

In summary, in this viewpoint article on computational modeling of elastic coefficients, we present a systematic approach in calculating elastic stiffness coefficients and bulk, shear, and Young's moduli of single crystals and multi-phases through first-principles calculations and CALPHAD modeling. The calculations are made for the binary $\mathrm{Mg}-\mathrm{Al}$ alloys and compared with the experimental data of AZ31 and AZ91 in the literature. At low temperatures, the predicted elastic coefficients agree well with experimental data. At high temperatures, both the dissolution of $\gamma-\mathrm{Al}_{12} \mathrm{Mg}_{17}$ and the grain boundary sliding affect the elastic properties of the alloys. Further works are needed in quantifying and separating their contributions.

\section{Acknowledgements}

The authors greatly appreciate financial support from NSF Grant 0510180, the US Automotive Materials Partnership through cooperative agreement No. DE-FC05-02OR22910, and US Army Research Laboratory for the grant W911NF-08-2-0064. The authors would also like to thank Shun Li Shang, Dong Eung Kim, and Arkapol Saengdeejing at The Pennsylvania State University for stimulating discussions and careful reading of the manuscript.

\section{References}

[1] G.B. Olson, Science 277 (1997) 1237-1242.

[2] Z.K. Liu, JOM 61 (10) (2009) 18-20.

[3] Z.K. Liu, L.Q. Chen, R. Raghavan, Q. Du, J.O. Sofo, S.A. Langer, C. Wolverton, J. Comput. Aided Mater. Des. 11 (2004) 183-199.

[4] Z.K. Liu, J. Phase Equilib. Diffus. 30 (2009) 517-534.

[5] R.S. Beals, Z.K. Liu, J.W. Jones, P.K. Mallick, D. Emadi, D. Schwam, B.R. Powell, JOM 59 (8) (2007) 43-48.
[6] S. Shang, H. Zhang, S. Ganeshan, Z.K. Liu, JOM 60 (12) (2008) 45-47.

[7] M. Mantina, Y. Wang, L.Q. Chen, Z.K. Liu, C. Wolverton, Acta Mater. 57 (2009) 4102-4108.

[8] S.L. Shang, Y. Wang, Z.K. Liu, Appl. Phys. Lett. 90 (2007) 101909.

[9] L. Kaufman, H. Bernstein, Computer Calculation of Phase Diagram, Academic Press Inc., New York, 1970.

[10] A.T. Dinsdale, CALPHAD 15 (1991) 317-425.

[11] J. Agren, J. Phys. Chem. Solids 43 (1982) 385-391.

[12] X.G. Lu, M. Selleby, B. Sundman, CALPHAD 29 (2005) 49-55.

[13] Y. Wang, S. Curtarolo, C. Jiang, R. Arroyave, T. Wang, G. Ceder, L.Q. Chen, Z.K. Liu, CALPHAD 28 (2004) 79-90.

[14] V. Ozolins, Phys. Rev. Lett. 102 (2009) 065702.

[15] S.L. Shang, A. Saengdeejing, Z.G. Mei, D.E. Kim, H. Zhang, S. Ganeshan, Y. Wang, Z.K. Liu, Comput. Mater. Sci., 2010, accepted for publication.

[16] Y.P. Varshni, Phys. Rev. B 2 (1970) 3952-3958.

[17] R. Lowrie, A.M. Gonas, J. Appl. Phys. 38 (1967) 45054509.

[18] O.D. Slagle, H.A. McKinstry, J. Appl. Phys. 38 (1967) 437-446.

[19] M. Hillert, J. Alloy. Compd. 320 (2001) 161-176.

[20] D. Kim, S.L. Shang, Z.K. Liu, Comput. Mater. Sci. 47 (2009) 254-260.

[21] R. Hill, Proc. Phys. Soc. Lond. A 65 (1952) 349-355.

[22] Z. Hashin, S. Shtrikman, J. Mech. Phys. Solids 10 (1962) 343-352.

[23] G.W. Milton, The Theory of Composites, Cambridge University Press, Cambridge, 2002.

[24] Z. Hashin, S. Shtrikman, J. Mech. Phys. Solids 11 (1963) 127-140.

[25] J.F. Nye, Physical Properties of Crystals: Their Representation by Tensors and Matrices, Clarendon Press, Oxford, 2000.

[26] Y. Wang, Z.K. Liu, L.Q. Chen, Acta Mater. 52 (2004) 2665-2671.

[27] H. Ledbetter, Mater. Sci. Eng. A Struct. Mater. Prop. Microstruct. Process. 442 (2006) 31-34.

[28] Y. Wang, J.J. Wang, H. Zhang, V.R. Manga, S.L. Shang, L.Q. Chen and Z.K. Liu, J. Phys.: Condens. Matter, submitted for publication.

[29] G.F. Davies, J. Phys. Chem. Solids 35 (1974) 1513-1520.

[30] A. Zunger, S.H. Wei, L.G. Ferreira, J.E. Bernard, Phys. Rev. Lett. 65 (1990) 353-356.

[31] C. Jiang, C. Wolverton, J. Sofo, L.Q. Chen, Z.K. Liu, Phys. Rev. B 69 (2004) 214202.

[32] S. Ganeshan, S.L. Shang, H. Zhang, Y. Wang, M. Mantina, Z.K. Liu, Intermetallics 17 (2009) 313-318.

[33] S. Ganeshan, S.L. Shang, Y. Wang, Z.K. Liu, Acta Mater. 57 (2009) 3876-3884.

[34] Y. Zhong, M. Yang, Z.K. Liu, CALPHAD 29 (2005) 303-311.

[35] H. Zhang, S.L. Shang, Y. Wang, A. Saengdeejing, L.Q. Chen and Z.K. Liu, Acta Mater., doi:10.1016/ j.actamat.2012.03.020.

[36] H. Watanabe, T. Mukai, M. Sugioka, K. Ishikawa, Scr. Mater. 51 (2004) 291-295.

[37] W. Sequeira, M.T. Murray, G.L. Dunlop, D.H. St. John, in: Proceedings of the TMS Symposium on Automotive Alloys, TMS, Warrendale, PA, 1997, pp. 169-183.

[38] Y. Carbonneau, M. Lepage, J. Renaud, D. Dube, in: E. Aghion, D. Eliezer (Eds.), Magnesium 2000, 2nd Israeli International Conference on Magnesium Science and Technology, Dead Sea, 2000, pp. 276-284.

[39] K. Ishikawa, H. Watanabe, T. Mukai, Mater. Lett. 59 (2005) 1511-1515. 